\title{
THE LIST EDGE COLORING AND LIST TOTAL COLORING OF PLANAR GRAPHS WITH MAXIMUM DEGREE AT LEAST 7
}

\author{
LiN Sun \\ School of Mathematics and Statistics \\ Lingnan Normal University \\ Guangdong Zhanjiang 524000, China \\ e-mail: fiona_sl@163.com \\ JIANLIANG WU \\ School of Mathematics, Shandong University \\ Jinan 250100, China \\ e-mail: jlwu@sdu.edu.cn \\ BING WANG ${ }^{1}$ \\ Department of Mathematics, Zaozhuang University \\ Shandong Zaozhuang, 27r160, China \\ e-mail: 2633122093@qq.com \\ AND \\ BIN LiU ${ }^{1}$ \\ Department of Mathematics, Ocean University of China \\ Qingdao 266100, China \\ e-mail: binliu@ouc.edu.cn.
}

\begin{abstract}
A graph $G$ is edge $k$-choosable (respectively, total $k$-choosable) if, whenever we are given a list $L(x)$ of colors with $|L(x)|=k$ for each $x \in E(G)$ $(x \in E(G) \cup V(G))$, we can choose a color from $L(x)$ for each element $x$ such that no two adjacent (or incident) elements receive the same color. The list edge chromatic index $\chi_{l}^{\prime}(G)$ (respectively, the list total chromatic
\end{abstract}

\footnotetext{
${ }^{1}$ Corresponding author.
} 
number $\chi_{l}^{\prime \prime}(G)$ ) of $G$ is the smallest integer $k$ such that $G$ is edge (respectively, total) $k$-choosable. In this paper, we focus on a planar graph $G$, with maximum degree $\Delta(G) \geq 7$ and with some structural restrictions, satisfies $\chi_{l}^{\prime}(G)=\Delta(G)$ and $\chi_{l}^{\prime \prime}(G)=\Delta(G)+1$.

Keywords: planar graph, list edge coloring, list total coloring.

2010 Mathematics Subject Classification: 05C15.

\section{REFERENCES}

[1] N. Alon and M. Tarsi, Colorings and orientation of graphs, Combinatorica 12 (1992) $125-134$. doi:10.1007/BF01204715

[2] N. Alon, Combinatorial Nullstellensatz, Combin. Probab. Comput. 8 (1999) 7-29. doi:10.1017/S0963548398003411

[3] M. Behzad, Graphs and Their Chromatic Numbers (Doctoral Thesis, Michigan State University, East Lansing, MI, 1965).

[4] J.A. Bondy and U.S.R. Murty, Graph Theory with Applications (MacMillan, London, 1976).

[5] M. Bonamy, Planar graphs with maximum degree $\Delta$ at least 8 are $(\Delta+1)$-edgechoosable, SIAM J. Discrete Math. 29 (2015) 1735-1763. doi:10.1137/130927449

[6] M. Bonamy, B. Lévêque and A. Pinlou, Planar graphs with $\Delta \geq 7$ and no triangle adjacent to $a C_{4}$ are minimally edge and total choosable, Discrete Math. Theoret. Comput. Sci. 17 (2016) 131-146. doi:10.1006/jctb.1997.1780

[7] O.V. Borodin, A.V. Kostochka and D.R. Woodall, List edge and list total colourings of multigraphs, J. Combin. Theory Ser. B 71 (1997) 184-204.

[8] O.V. Borodin, A generalization of Kotzig's theorem on prescribed edge coloring of planar graphs, Mat. Zametki 48 (1990) 22-28, in Russian.

[9] A.J. Harris, Problems and Conjectures in Extremal Graph Theory, Ph. D. Dissertation (Cambridge, Cambridge University, 1984).

[10] J.F. Hou, G.Z. Liu and J.S. Cai, List edge and list total colorings of planar graphs without 4-cycles, Theoret. Comput. Sci. 369 (2006) 250-255.

doi:10.1016/j.tcs.2006.08.043

[11] J.F. Hou, G.Z. Liu and J.L. Wu, Some results on list total colorings of planar graphs, Lecture Notes in Comput. Sci. 4489 (2007) 320-328. doi:10.1007/978-3-540-72588-6_53

[12] T.R. Jensen and B. Toft, Graph Coloring Problems (Wiley Interscience, New York, 1995). 
[13] M. Juvan, B. Mohar and R. Škrekovski, Graphs of degree 4 are 5-edge-choosable, J. Graph Theory 32 (1999) 250-262. doi:10.1002/(SICI)1097-0118(199911)32:3〈250::AID-JGT5〉3.0.CO;2-R

[14] M. Juvan, B. Mohar and R. Škrekovski, List total colourings of graphs, Combin. Probab. Comput. 7 (1998) 181-188. doi:10.1017/S0963548397003210

[15] Q.L. Lu, Z.K. Miao and Y.Q. Wang, Sufficient conditions for a planar graph to be list edge $\Delta$-colorable and list totally $(\Delta+1)$-colorable, Discrete Math. 313 (2013) 575-580. doi:10.1016/j.disc.2012.11.026

[16] V.G. Vizing, Some unsolved problems in graph theory, Uspekhi Mat. Nauk 23 (1968) 117-134, in Russian. doi:10.1070/RM1968v023n06ABEH001252

[17] W.F. Wang and K.W. Lih, Choosability, edge choosability, and total choosability of outerplane graphs, European J. Combin. 22 (2001) 71-78. doi:10.1006/eujc.2000.0430

[18] H.J. Wang, L.D. Wu, X. Zhang, W.L. Wu and B. Liu, A note on the minimum number of choosability of planar graphs, J. Comb. Optim. 31 (2016) 1013-1022. doi:10.1007/s10878-014-9805-2

[19] H.J. Wang, B. Liu, X. Zhang, L.D. Wu, W.L. Wu and H.W. Gao, List edge and list total coloring of planar graphs with maximum degree 8, J. Comb. Optim. 32 (2016) 188-197. doi:10.1007/s10878-015-9870-1

[20] J.L. Wu and P. Wang, List-edge and list-total colorings of graphs embedded on hyperbolic surfaces, Discrete Math. 308 (2008) 6210-6215. doi:10.1016/j.disc.2007.11.044

Received 13 February 2017

Revised 2 June 2018 Accepted 21 June 2018

\section{APPENDIX}

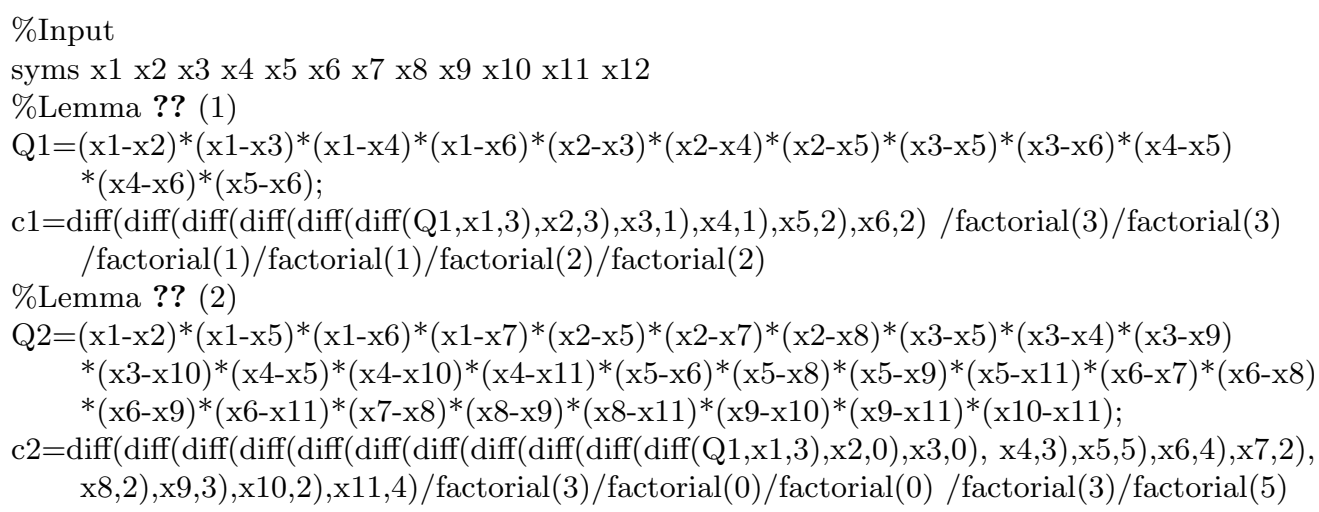


/factorial(4)/factorial(2)/factorial(2)/factorial(3)/factorial(2)/factorial(4)

\%Lemma ?? (3)

$\mathrm{Q} 3=(\mathrm{x} 1-\mathrm{x} 2)^{*}(\mathrm{x} 1-\mathrm{x} 3)^{*}(\mathrm{x} 1-\mathrm{x} 4)^{*}(\mathrm{x} 1-\mathrm{x} 5)^{*}(\mathrm{x} 1-\mathrm{x} 9)^{*}(\mathrm{x} 1-\mathrm{x} 8)^{*}(\mathrm{x} 2-\mathrm{x} 3)^{*}(\mathrm{x} 2-\mathrm{x} 5)^{*}(\mathrm{x} 2-\mathrm{x} 6)^{*}(\mathrm{x} 3-\mathrm{x} 4)$ $*(\mathrm{x} 3-\mathrm{x} 6)^{*}(\mathrm{x} 3-\mathrm{x} 9)^{*}(\mathrm{x} 3-\mathrm{x} 7) *(\mathrm{x} 4-\mathrm{x} 7) *(\mathrm{x} 4-\mathrm{x} 8)^{*}(\mathrm{x} 5-\mathrm{x} 9) *(\mathrm{x} 5-\mathrm{x} 6) *(\mathrm{x} 5-\mathrm{x} 8)^{*}(\mathrm{x} 6-\mathrm{x} 7) *(\mathrm{x} 6-\mathrm{x} 9)$ $*(\mathrm{x} 7-\mathrm{x} 8) *(\mathrm{x} 7-\mathrm{x} 9) *(\mathrm{x} 8-\mathrm{x} 9)$;

$\mathrm{c} 3=\operatorname{diff}(\operatorname{diff}(\operatorname{diff}(\operatorname{diff}(\operatorname{diff}(\operatorname{diff}(\operatorname{diff}(\operatorname{diff}(\operatorname{diff}(\mathrm{Q} 1, \mathrm{x} 1,3), \mathrm{x} 2,3), \mathrm{x} 3,3), \mathrm{x} 4,3), \mathrm{x} 5,3), \mathrm{x} 6,3), \mathrm{x} 7,3)$, $\mathrm{x} 8,2), \mathrm{x} 9,0) /$ factorial $(3) /$ factorial $(3) /$ factorial(3)/factorial(3)/factorial(3)

/factorial(3)/factorial(3)/factorial(2) 